\title{
Apple cultivar effects on codling moth (Lepidoptera: Tortricidae) egg mortality following fumigation with methyl bromide
}

\author{
John H. Maindonald ${ }^{\mathrm{a}, *}$, Barbara C. Waddell ${ }^{\mathrm{b}}$, Robert J. Petry ${ }^{\mathrm{b}}$ \\ a Australian National University, Canberra ACT 0200, Australia \\ b The Horticulture and Food Research Institute of New Zealand Limited, Private Bag 92 169, Auckland, New Zealand
}

Received 27 April 2000; accepted 17 December 2000

\begin{abstract}
Fumigation experiments with methyl bromide (MeBr) were carried out in New Zealand over several seasons. In 1988-1989 'Braeburn', 'Fuji', 'Granny Smith', 'Red Delicious', 'Royal Gala', 'Gala' and 'Splendour' apples were infested with freshly laid eggs of codling moth, Cydia pomonella (L), and were fumigated at a range of methyl bromide doses. The concentration-time $\mathrm{ct}_{\text {sum }}$, which estimates the average methyl bromide concentration over the 120 min of fumigation, was used as the measure of exposure. The complementary log-log of mortality induced by treatment varied linearly with the $\mathrm{ct}_{\text {sum }}$, with the same equation for all apple cultivars. This relationship allowed accurate prediction of a $99 \%$ lethal value $\left(\mathrm{LC}_{99}\right)$ for the $\mathrm{ct}_{\text {sum }}$ that was again, the same for all cultivars. Subsequent experiments in 1998-1989 with 'Red Delicious', 'Fuji' and 'Pacific Rose' apples' were conducted in the absence of codling moth eggs with the aim of examining the regression of $\mathrm{ct}_{\mathrm{sum}}$ on dose. There were different lines for different cultivars, and different intercepts for different years. The $\mathrm{ct}_{\text {sum }}$ estimates, at an injected dose of $24 \mathrm{~g} \mathrm{~m}^{-3}$ could not, however, be distinguished between cultivars. The estimate of $\mathrm{ct}_{\text {sum }}$ for 'Pacific Rose' at $24 \mathrm{~g} \mathrm{~m}^{-3}$ cannot be distinguished from the $\mathrm{ct}_{\text {sum }}$ estimates for cultivars currently approved for export to Japan. The approved treatment for New Zealand apples exported to Japan includes an injected methyl bromide dose of $24 \mathrm{~g} \mathrm{~m}^{-3}$. For acceptance of this treatment for a new variety, it is sufficient to demonstrate that the $\mathrm{ct}_{\text {sum }}$ is maintained at levels which lie within or above the range found for existing approved varieties. (c) 2001 Elsevier Science B.V. All rights reserved.
\end{abstract}

Keywords: Insecta; Cydia pomonella; Disinfestation

* Corresponding author. Tel.: + 61-2-61253998; fax: + 612-6125549.

E-mail address: john.maindonald@anu.edu.au (J.H. Maindonald).

\section{Introduction}

Cultivars of all fruits have historically been treated as different entities, with an identical extensive testing protocol applied to each, to verify the efficacy of a defined postharvest treatment aimed at disinfesting codling moth Cydia 
pomonella (L.). Similarities in response to methyl bromide (MeBr) have been reported between cultivars of a given fruit (Tebbets et al., 1983; Yokoyama et al., 1990; Maindonald et al., 1992) thus challenging the requirement for cultivar by cultivar testing. Although effort is going into the development of alternative treatments, further new varieties are being evaluated for their export potential using $\mathrm{MeBr}$ technology. Effective alternatives are not widely available and exemptions currently apply for quarantine and pre-shipment uses, thus maintaining the efforts in $\mathrm{MeBr}$ research and raising the question of varietal differences.

Maindonald et al. (1992) investigated the relationship between codling moth mortality and $\mathrm{MeBr}$ dose for six cherry cultivars to determine, if cultivars could be considered the same or if, they were different. The study investigated cultivar and seasonal differences for cherries exported from New Zealand to Japan. The study found that $\mathrm{MeBr}$ concentration decreases with time because of adsorption by the fruit, at a rate which may vary from one cultivar to another (up to $30 \%$ of the chamber gas can be absorbed during a $2 \mathrm{~h}$ fumigation). The concentration-time sum $\left(\mathrm{ct}_{\text {sum }}\right)$ is the product of the time and an estimate of the average concentration. The results showed variation between cultivars in the $\mathrm{ct}_{\text {sum }}$ at a given injected dose, with a relationship between mortality and $\mathrm{ct}_{\text {sum }}$ that was independent of dose. The fruit absorption determined the relationship between $\mathrm{ct}_{\text {sum }}$ and dosage but it was the $\mathrm{ct}_{\text {sum }}$ that affected mortality irrespective of any one cultivar's individual absorption properties. This implies that the testing of the relationship between $\mathrm{ct}_{\text {sum }}$ and injected dose for a new cultivar is enough to establish that an approved treatment will be effective when used with a cultivar where there have been no studies with live insects.

The current paper draws on and modifies the methods developed for the cherry study. A (nonlinear) relationship was established between the logit of codling moth mortality and $\mathrm{ct}_{\text {sum. This }}$ determined the $\mathrm{ct}_{\text {sum }}$ that will kill $99 \%$ of insects; we call this the $\mathrm{LC}_{99}$ or $99 \%$ lethal concentration. The same measure of $\mathrm{MeBr}$ concentration has been used in the present investigation, where the aims were to (1) define the relationship between codling moth mortality and $\mathrm{ct}_{\text {sum }}$ for approved export apple cultivars; (2) characterise the $\mathrm{MeBr}$ absorption as a function of injected $\mathrm{MeBr}$ dose for the different apple cultivars; and (3) compare a new cultivar, 'Pacific Rose', with approved cultivars to determine, if the approved treatment would be effective against codling moth eggs, potentially infesting this new export candidate cultivar.

\section{Materials and methods}

Research was conducted in two decades. The 1980s research used codling moth infested apples. In 1988, the cultivars were 'Red Delicious' (three replicates), 'Fuji', 'Granny Smith', 'Braeburn' and 'Royal Gala' (two replicates per cultivar). In 1989, 'Red Delicious', 'Gala' and 'Splendor' apples were infested and fumigated (two replicates per cultivar). The research used 78649 eggs in 1988 and 55175 eggs in 1989. An average of 7872 eggs were used per replicate.

In the 1990s, a total of three cultivars were evaluated in the absence of codling moth eggs. 'Red Delicious' and 'Fuji' were investigated in 1998 (three replicates) and again in 1999 together with a new apple cultivar, 'Pacific Rose' (two replicates per cultivar).

\subsection{Fumigation procedure}

Fumigations were carried out in 23.41 Plexiglass chambers using methods described earlier (Maindonald et al., 1992). Each chamber was loaded with $3.2 \mathrm{~kg}$ of fruit of one cultivar. The external dimensions of the fruit load (including spaces between fruits) occupied approximately $40 \%$ of the total volume of the chamber and associated ducting. The $2 \mathrm{~h}$ fumigation was timed from the end of the gas injection and was conducted at $12.0 \pm 0.5^{\circ} \mathrm{C}$. $\mathrm{MeBr}$ concentrations in each chamber were sampled throughout the fumigation and analysed by gas chromatography, in most instances at 5, 30, 60, 90 and 120 min from the start of fumigation. For use as a standard, an empty chamber was injected with $30 \mathrm{~g} \mathrm{~m}^{-3}$ and 
sampled at the same time as each treatment chamber. This standard was used to calibrate the concentration in the treatment chambers.

In all studies, each replicate provided results for one apple cultivar at each of six doses of $\mathrm{MeBr}$. Except for 'Royal Gala', the doses were 0, 8, 12, $16,20,24$ and $30 \mathrm{~g} \mathrm{~m}^{-3}$. 'Royal Gala' used a dose range which included 5 and excluded $30 \mathrm{~g}$ $\mathrm{m}^{-3}$. A total of 29 replicates were fumigated.

Each replicate in 1988 and 1989 provided the following data for each injected dose: (1) insect mortality at the injected dose $\mathrm{MeBr}$; and (2) concentration $c_{t}$, at each of the time points $t=5,10$, 30, 60, 90, $120 \mathrm{~min}$. Note the distinction between the initially injected dose and subsequent measurements of $\mathrm{MeBr}$ concentration in the chamber during the course of the fumigation. The concentration-time sum (in $\mathrm{g} \mathrm{h} \mathrm{m}^{-3}$ ) was estimated (Maindonald et al., 1992) as

$$
\begin{aligned}
\mathrm{ct}_{\mathrm{sum}}= & \left(7.5 \times c_{5}+12.5 \times c_{10}+25 \times c_{30}+30 \times c_{60}\right. \\
& \left.+30 \times c_{90}+15 \times c_{120}\right) / 60
\end{aligned}
$$

This is a simplification of the formula obtained from summing, over each interval between concentration measurements, the product of average concentration and the length of the time interval. Replicates in 1998-1999 provided data for concentration only. The lethal dose resulting in 50 and $99 \%$ mortality $\left(\mathrm{LD}_{50}\right.$, and $\mathrm{LD}_{99}$, respectively) relate to injected dose and are measured as $\mathrm{g} \mathrm{m}^{-3}$ of the empty chamber. The $\mathrm{LC}_{50}$ and $\mathrm{LC}_{99}$ refer to the $\mathrm{ct}_{\text {sum }}$ and are measured as $\mathrm{g} \mathrm{h} \mathrm{m}^{-3}$.

\subsection{Source of apples and insects}

All fruit was harvested in Hawke's Bay or Nelson, was count size 100 (174-199 g) and was not waxed. Fruit intended for infestation was free of insecticide sprays and was harvested and packed from the HortResearch research orchard network. Fruit obtained from commercial growers had undergone a conventional export spray programme and postharvest handling i.e. washed, graded (including washed and sorted by size), and packed. Fruit was stored at $0.5^{\circ} \mathrm{C}$ for up to 8 weeks prior to treatment.

Codling moth were from a laboratory colony maintained on artificial diet using rearing condi- tions and methods identical to those described by Maindonald et al. (1992). The colony was established in 1967 and annual introductions of fieldcollected adults were made until 1975. The insects used in 1988 were from generations 172 to 174 and in 1989 from generations 182 to 183 . Adult moths were allowed to oviposit on apples for $24 \mathrm{~h}$ at $25 \pm 0.5^{\circ} \mathrm{C}$. The moths were then removed and the apples carrying the freshly laid eggs were stored at $12.0 \pm 1{ }^{\circ} \mathrm{C}$ in preparation for fumigation the following morning. An untreated (control) group of infested fruit was kept for each replicate to determine natural codling moth mortality.

After fumigation the infested fruit were removed from the chambers and held for 9 days at $25.0 \pm 0.5^{\circ} \mathrm{C}$, until mortality could be determined by the presence or absence of hatching in both treated and control eggs.

\subsection{Statistical analysis}

\subsubsection{Insect mortality}

Codling moth mortality data were available from 11 replicates treated in 1988 and six replicates in 1989 . Let $p^{*}$ be the mortality and let $c$ be the control mortality. We investigated several alternative mortality response curves. After checking and comparing these, we chose a transformation that gave a response that was very nearly linear with changes in $\mathrm{ct}_{\text {sum. }}$. The response $y$ values were determined as

$y=\log (-\log (1-p))$, where $p=\frac{p^{*}-c}{1-c}$

Values of $y$ then became the dependent variable in a regression model that we now describe. The model was

$y_{i j}=a_{0}+a_{i}+\left(b_{0}+b_{i}\right) x_{i j}+e_{i j}$

where $j$ indexes the replicate, $i$ indexes the $i$ th dose within the replicate, $x_{i j}$ is the $\mathrm{ct}_{\mathrm{sum}}, a_{i}$ is a random (between replicates) intercept term, $b_{i}$ is a random (between replicates) slope term and $e_{i j}$ is the variation of the $j$ th point about the line for the $i$ th replicate. We investigated the use of different lines (different $a_{0}$ and $b_{0}$ ) for different cultivars and different years. 
The analysis procedure was an extension of weighted multiple regression, using $y$ as the dependent variable, but allowing for a random change in slope between replicates. Iteration was necessary to determine the appropriate weights because the estimate of $\operatorname{var}[y]$ is not constant, but depends at each point on the expected values of $p$ and of $c$. Methods of this type are described in Chapter 3 of Finney (1971). The method is an approximation to maximum likelihood, rather than true maximum likelihood. The method can be expected to work well when the numbers of insects at each dose in each replicate are large, in the present data between 239 and 2965.

Calculations proceed iteratively, with the weights for the current iteration taken as the inverses of the variance estimates that are obtained using fitted values of $p$, determined by back-calculation from Eq. (1), from the earlier iteration. Iteration ceased when there was no further change to the weights. Working from the first order Taylor series expansion of $\log \left(\left(p^{*}-\mathrm{c}\right) /(1-\right.$ c)) about the expected values $E\left[p^{*}\right]$ and $E[c]$ we find that

$\operatorname{var}[p] \approx \frac{1}{(1-E[c])^{2}} \operatorname{var}\left[p^{*}\right]+\frac{\left(1-E\left[p^{*}\right]\right)^{2}}{(1-E[c])^{2}} \operatorname{var}[c]$

where

$$
\begin{aligned}
\operatorname{var}\left[p^{*}\right] & =\frac{\phi E\left[p^{*}\right]\left(1-E\left[p^{*}\right]\right)}{n}, \text { and } \operatorname{var}[c] \\
& =\frac{\phi E[c](1-E[c])}{n_{c}}
\end{aligned}
$$

The estimate of $p^{*}$ is based on $n$ insects, while the estimate of $c$ is based on $n_{c}$ insects. The first iteration of the fitting procedure uses $p^{*}$ as an estimate of $E\left[p^{*}\right]$. The variances are those for a binomial distribution, but inflated by a heterogeneity factor $\phi$. Then, with a further use of a first order Taylor series expansion

$\operatorname{var}[y] \approx \frac{1}{(1-E[p])^{2}[\log (1-E[p])]^{2}} \operatorname{var}[p]$.

The iterative reweighting is analogous to that used for Generalised Linear Model (GLM) calculations (McCullagh and Nelder, 1989). The ad- justment for control mortality does not fit within the GLM framework.

The linear mixed effects (lme) abilities from S-PLUS 2000 were used for the main part of the calculations. This allowed modelling of a random replicate to replicate variation in the slope of the response (Longford 1993), an ability that is not available in conventional software for bio-assay analysis. It also allowed modelling of a sequential correlation structure. Variation about the fitted response may be positively correlated for neighbouring dose levels.

Residuals were checked at each step. Observations whose normalised residuals were greater than 2.6 in absolute value, corresponding to a nominal $1 \%$ two-sided critical value in a normal distribution, were treated as outliers. Such outliers were omitted when testing for cultivar and season effects, as they have the potential to bias results. Details of such omissions are reported.

Plots of normalised residuals against fitted values were used to check whether the variances followed Eq. (5). Residuals are normalised by dividing by their standard errors. The standard deviation (S.D.) that we used, took into account both Eq. (5) and replicate variation. A specific concern was to determine whether the scale parameter $\phi$ in Eq. (4) is constant.

\section{4. $\mathrm{MeBr}$ sorption data}

Multiple regression was used to derive a prediction equation, using all data where there was a complete set of $\mathrm{MeBr}$ concentrations (i.e. 6 readings over the course of the $2 \mathrm{~h}$ fumigation), for the 5-min concentration given concentrations at remaining time points. The equation was used to estimate four missing 5-min concentrations in the 1988 data. A similar procedure was followed for estimating one missing 120-min concentration in the 1999 data.

The lme model abilities from S-PLUS 2000 were used to determine an equation for predicting, for each cultivar separately, the $\mathrm{ct}_{\text {sum }}$ at each injected dose. Possible year effects were investigated. The linear mixed effects library feature of the software was used to model random betweenreplicate and within-year slope effects. 
The mean deviance, obtained by taking twice the difference between the log-likelihood for the fitted model and the log-likelihood for a model that fits the data exactly, then dividing by the difference in the degrees of freedom, provides a measure of the lack of fit. The deviance is comparable with the residual sum of squares in the usual form of regression calculations. Degrees of freedom are determined by summing over the degrees of freedom for each of the response curves. With the binomial error model, the theoretical value for the mean deviance (in any individual replicate or overall) is 1.0. With data such as these, additional sources of variation usually result in a mean deviance that is substantially $>1.0$ (Maindonald, 1984 , p. 243-246). This mean deviance is an estimate of $\phi$ in Eq. (4). Sources of variation that may affect results include the insect material, fruit sorption, and measurement and circulation of fumigant within the chambers.

\section{Results}

\subsection{Codling moth mortality response on different apple cultivars}

Fig. 1 examines the probit form of response that has often been assumed for $\mathrm{MeBr}$ mortality data. The response curve in Fig. 1 is clearly not a straight line, as it would be if the response were probit. By contrast the complementary $\log -\log$ response (Fig. 2) gave a good fit to the data. A model in which the treatment-adjusted mortality depended only on the $\mathrm{ct}_{\text {sum }}$ was adequate. Based on the increase in the log-likelihood, there was no reason to use either a model which allowed different intercepts and slopes $\left(X^{2}=12.7,16\right.$ d.f., $p=$ 0.39 ) for different cultivars, or a model which allowed different intercepts for different years $\left(X^{2}=0.45,1\right.$ d.f., $\left.p=0.50\right)$.

Six data points were omitted in the process of fitting the data. The method of analysis was not

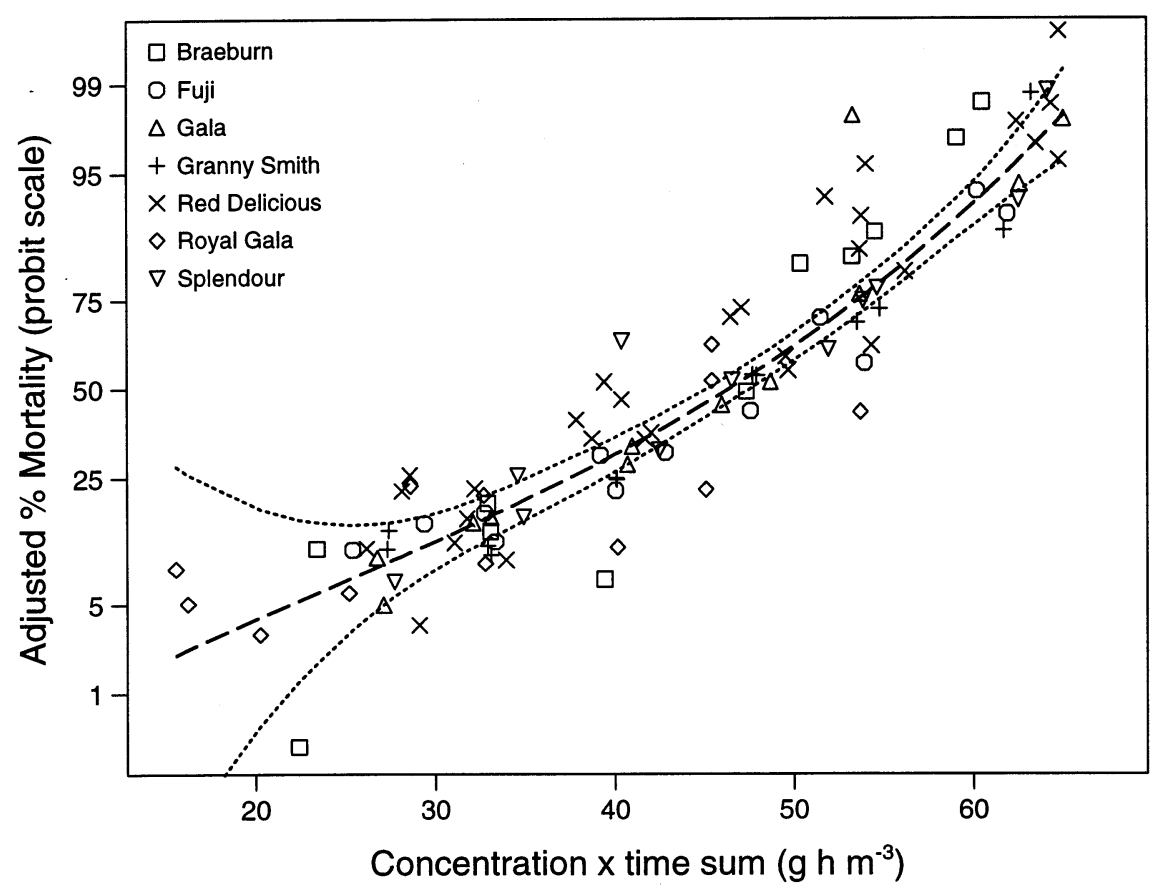

Fig. 1. Plot of adjusted mortality (probit scale) versus concentration-time sum ( $\mathrm{ct}_{\text {sum }}$ ). The dashed line shows a fitted spline smooth, with $95 \%$ pointwise confidence bounds shown as dotted lines. The confidence bounds indicate that the fitted spline smooth is inconsistent with fitting a line. 


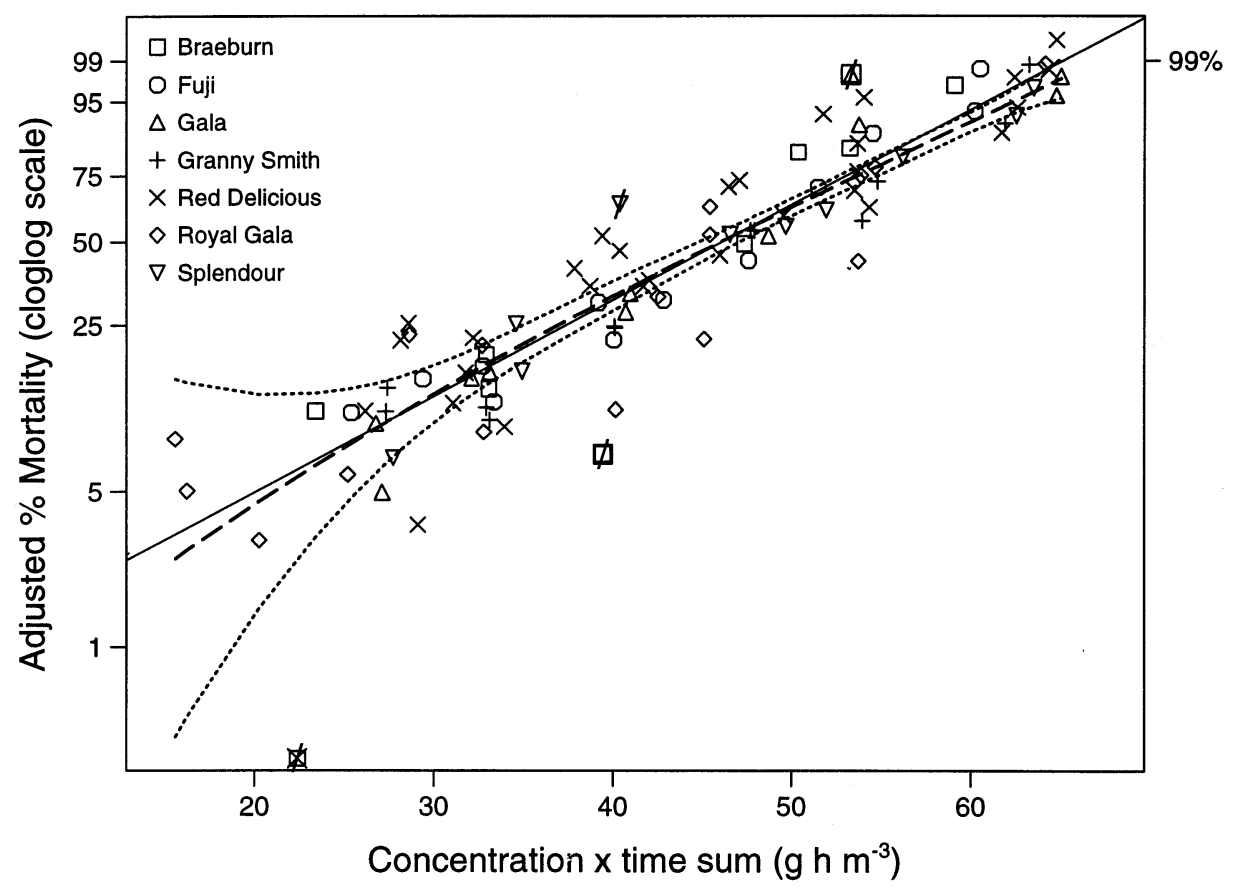

Fig. 2. Plot of adjusted mortality (complementary log-log scale) versus concentration-time sum. The dashed line shows a fitted spline smooth, with $95 \%$ pointwise confidence bounds shown as dotted lines. Points that are identified as outliers are shown with a $\operatorname{slash}(/)$.

able to use points where the observed mortality was less than the control mortality, which ranged from 15.1 to $27.7 \%$. This led to the omission of three points ('Braeburn' at $12 \mathrm{~g} \mathrm{~m}^{-3}$ in 1988, and 'Red Delicious' and 'Splendour' at $8 \mathrm{~g} \mathrm{~m}^{-3}$ in 1989). Four further points were omitted according to the criterion noted in the methods section, which identified the points as outliers. These were for 'Braeburn' at 8 and $16 \mathrm{~g} \mathrm{~m}^{-3}$ in 1988, 'Gala' at $24 \mathrm{~g} \mathrm{~m}^{-3}$ and 'Splendour' at 8 and $16 \mathrm{~g} \mathrm{~m}^{-3}$ (both in 1989).

\subsection{Prediction of mortality as a function of $c t_{\text {sum }}$}

The fitted equation was

$y=-4.98[\mathrm{SE}=0.13]+0.0998[\mathrm{SE} 0.0030] x$

where $x=\mathrm{ct}_{\text {sum }}$ and $y=\log (-\log (1-p))$. Recall that $p=\left(p^{*}-c\right) /(1-c)$.

Fig. 2 shows values of $y$, plotted against the $\mathrm{ct}_{\text {sum }}$, with the different cultivars identified separately. Also superimposed is a smooth curve that has been fitted using a spline procedure (MathSoft, 1999), together with 95\% confidence bounds for this smooth fitted curve. The confidence bounds are wide enough, especially at low and high values of $\mathrm{ct}_{\text {sum }}$, that the fitted spline smooth is consistent with fitting a line.

Fig. 3 shows how the weights of points changed with the expected treatment induced mortality. Control mortalities and numbers of eggs will typically be different at different points with the same treatment induced mortality, leading to the different weights that are apparent in this plot.

As a check that the weighting function (determined by Eq. (4)) is working correctly, Fig. 4 presents a plot of normalised residuals against $\log (-\log (1-\hat{p}))$, where $\hat{p}$ is the estimated mortality due to treatment. This plot shows no evident trend or other pattern. 
3.3. Methyl bromide sorption by different apple cultivars

Mean values of the $\mathrm{ct}_{\text {sum }}$ for different $\mathrm{MeBr}$ doses are given in Table 1. Different lines for the different cultivars were fitted (Fig. 5). The slopes were different between cultivars (Table 2). In fitting the lines, four points were omitted according to the criterion noted in the methods section, which identified the points as outliers. They were for; 'Royal Gala' in 1988 at $24 \mathrm{~g} \mathrm{~m}^{-3}$, 'Braeburn' in 1988 at $20 \mathrm{~g} \mathrm{~m}^{-3}$, 'Red Delicious' in 1988 at $20 \mathrm{~g} \mathrm{~m}^{-3}$, and 'Fuji' in 1999 at $16 \mathrm{~g} \mathrm{~m}^{-3}$. The first was negative, and the remaining three were positive.

Table 3 compares the predicted values for the $\mathrm{ct}_{\text {sum }}$ when the $\mathrm{MeBr}$ dose was $24 \mathrm{~g} \mathrm{~m}^{-3}$. The confidence intervals all overlap. A multiple comparison test $(p=0.05)$ does not distinguish them. The estimates fall into two groups. 'Pacific Rose', 'Fuji', 'Granny Smith' and 'Red Delicious' comprise one group, where the $\mathrm{ct}_{\text {sum }}$ is relatively higher than that of the remaining four cultivars. 'Braeburn', 'Royal Gala', 'Gala' and 'Splendour' com- prise the other group. Our particular interest is in the comparison of 'Pacific Rose', for which mortality data are not available, with other apple cultivars. 'Pacific Rose' belongs to the group where the $\mathrm{ct}_{\text {sum }}$ is relatively higher. A MeBr dose of $24 \mathrm{~g} \mathrm{~m}^{-3}$ will lead to a $\mathrm{ct}_{\text {sum }}$ that is among the four largest predicted values. Recall that mortalities will be high, independently of cultivar, when the $\mathrm{ct}_{\text {sum }}$ is large.

\subsection{The effect of outliers}

The change in estimates when outliers are included was checked. The equation for the regression of logit(adjusted mortality) on $\mathrm{ct}_{\text {sum }}$ changed little $(-4.95+0.0992 x$ in place of $-4.97+$ $0.0998 x$ ). Standard errors for the intercept and slope increased by 50 and $30 \%$, respectively. Changes in estimates and confidence intervals for the $\mathrm{LC}_{50}$ and the $\mathrm{LC}_{99}$ were inconsequential, less than 0.1 in each case. The maximum change in any of the lethal dose estimates in Table 4 was 0.4 . Some confidence intervals increased in width by comparable amounts.

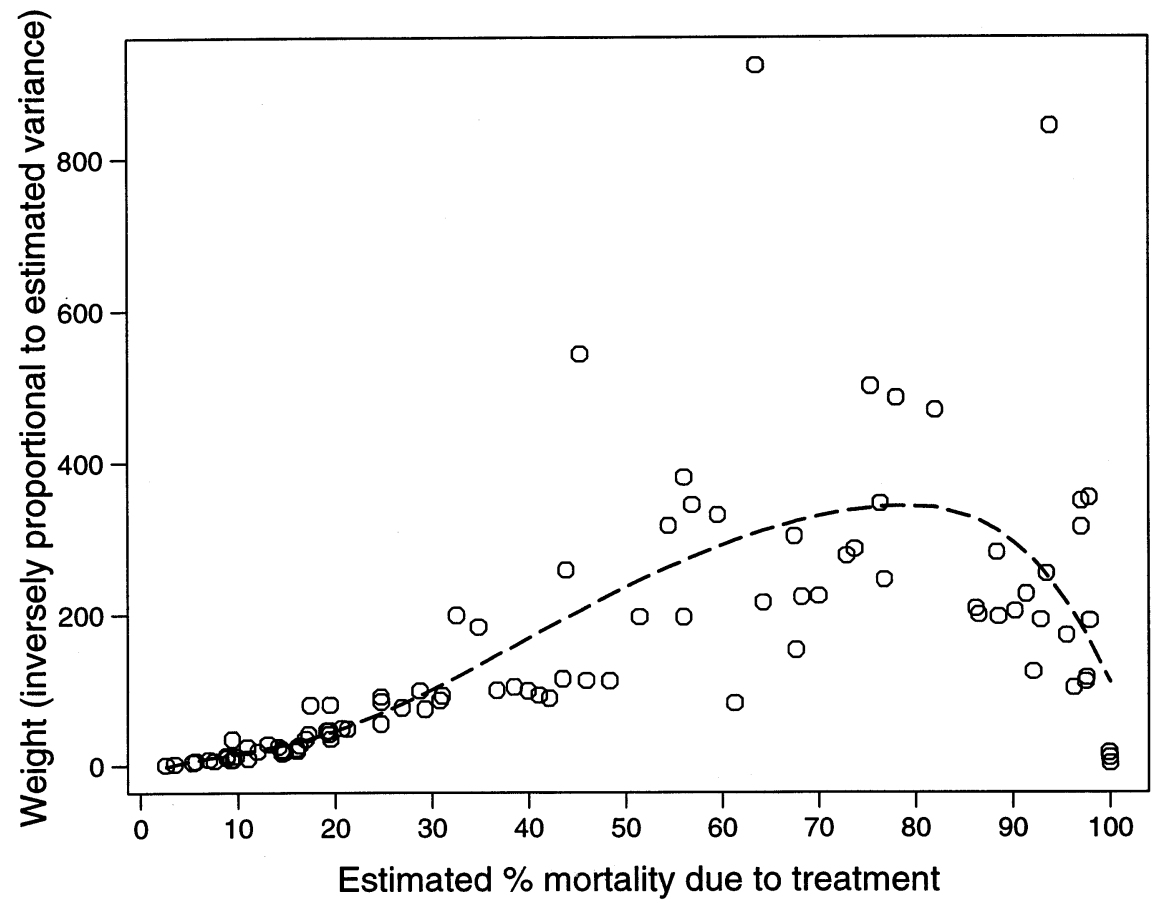

Fig. 3. Variation in weight assigned to observations with estimated mortality due to treatment. 


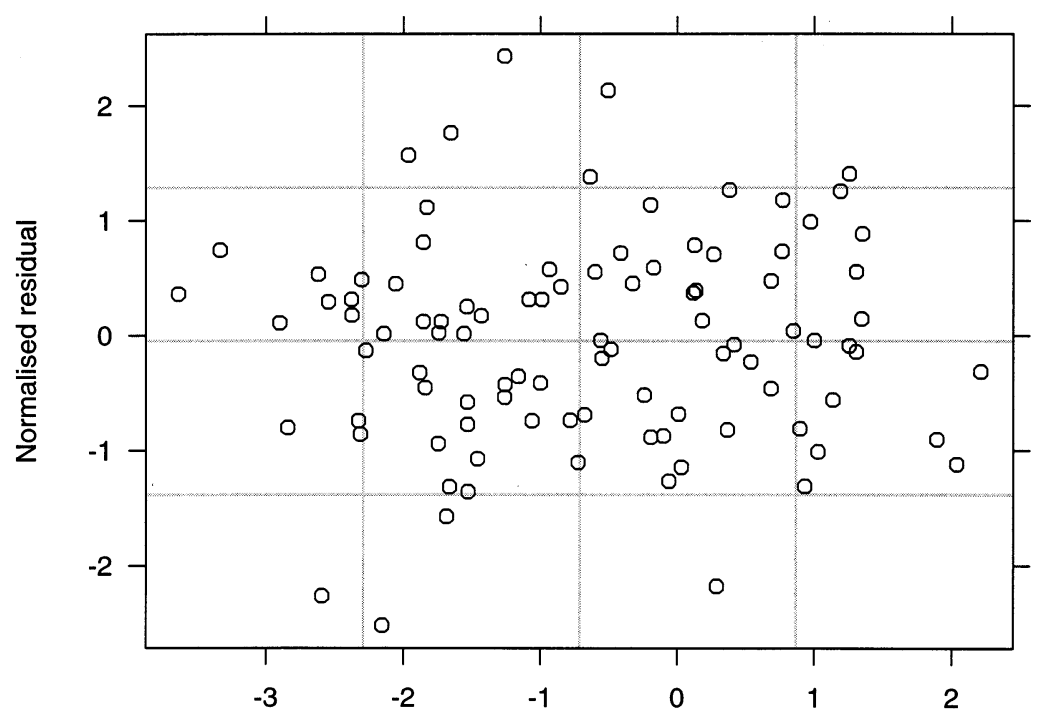

Complementary log-log transform of estimated mortality due to treatment

Fig. 4. Normalised residuals, i.e. divided by estimates of their standard error, plotted against fitted values on a complementary $\log -\log$ scale. If the choice of weights (Fig. 3) is correct, normalised residuals should show approximately constant scatter.

\section{Discussion and conclusions}

The pattern of response in the tolerance distribution is consistent with that found for codling moth infesting cherries (Maindonald et al., 1992) where, to a close approximation

logit (adjusted mortality) $=a+b\left(\text { ct }_{\text {sum }}\right)^{2}$.

For the range of codling moth mortalities in the data examined, both in the present paper and in the 1992 paper, this equation predicts mortalities that are almost identical to those predicted by the equation used here, i.e. by

$\log (-\log (1-$ adjusted mortality $))$

$=a_{0}+b_{0}\left(\mathrm{ct}_{\text {sum }}\right)$.

The complementary $\log -\log$ tolerance distribution can be motivated from a connection with an extreme value distribution. The insect dies when the first of a number of its life maintenance 'systems' fails. Details of the argument are included in the Appendix A.

Fig. 3 highlights the large changes in variance with change in the estimated mortality due to treatment. It is important to weight the points correctly. If this is not done, variation in mortality at low mortalities, which are due to random variation, is likely to be interpreted as requiring different fitted lines in Fig. 2.

The $\mathrm{LC}_{99}$ of 65.2 (95\% C.I. 62.9-67.7) for the $\mathrm{ct}_{\text {sum }}$ for these apple cultivars should be compared with the figure of 87.1 [SE 0.81] that Maindonald et al. (1992) found for the use of MeBr to disinfest

Table 1

Mean values of the $\mathrm{ct}_{\text {sum }}$ for the different doses, for apple cultivars fumigated with methyl bromide at a range of nominal doses for $2 \mathrm{~h}$ at $12.0 \pm 0.5^{\circ} \mathrm{C}^{\mathrm{a}}$

$\begin{array}{lllll}\text { Dose } \mathrm{D}, \mathrm{g} \mathrm{m}^{-3} & \mathrm{ct}_{\text {sum }} & c_{5} & c_{90} & \begin{array}{l}\text { Number of } \\ \text { points }\end{array}\end{array}$

\begin{tabular}{rrrrr}
\hline 5 & 15.93 & 8.05 & 8.13 & 2 \\
8 & 25.17 & 12.75 & 13.03 & 32 \\
12 & 32.24 & 15.88 & 16.19 & 32 \\
16 & 40.48 & 20.46 & 19.79 & 32 \\
20 & 47.31 & 24.13 & 23.30 & 31 \\
23 & 54.66 & 28.02 & 28.06 & 1 \\
24 & 53.71 & 27.91 & 26.51 & 32 \\
30 & 63.90 & 33.00 & 31.38 & 30 \\
\hline
\end{tabular}

a In the majority of replicates the doses were 8, 12, 16, 20, 24 and 30 . 


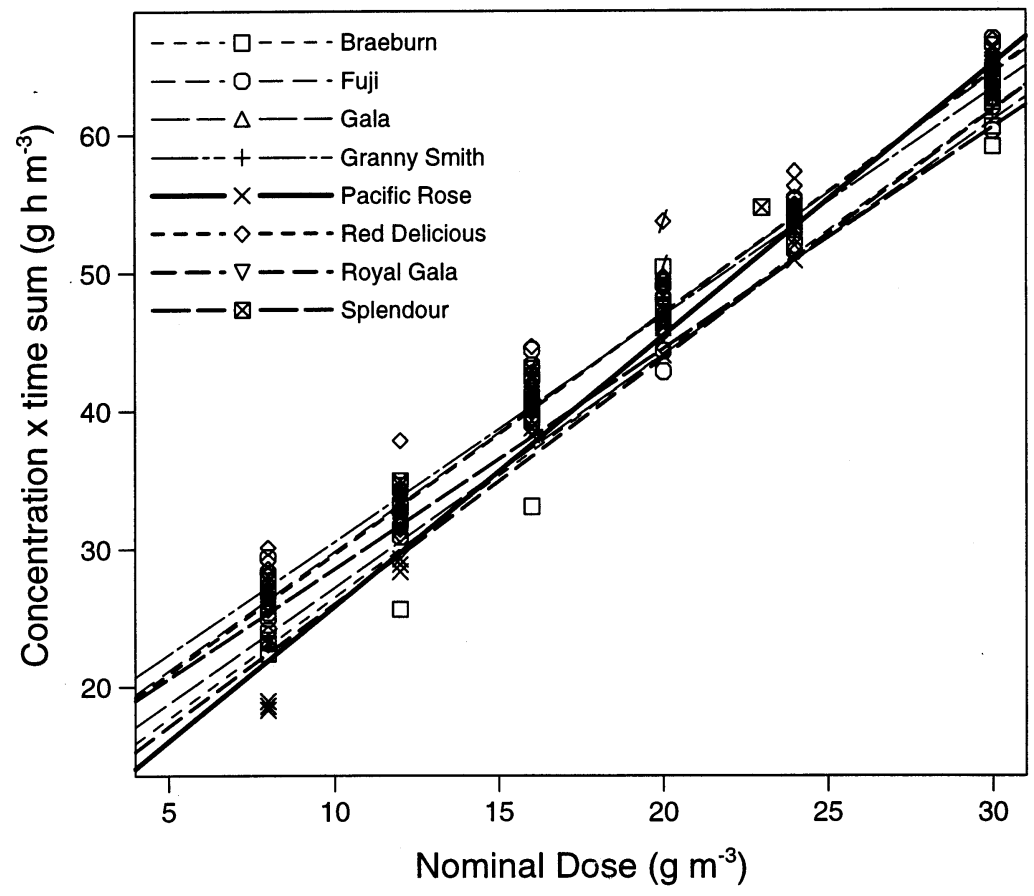

Fig. 5. Plot of $\mathrm{ct}_{\text {sum }}$ against nominal dose. The graph shows different lines for the different cultivars. Points that are identified as outliers are shown with a slash (/).

codling moth from cherries. Losses due to sorption were much greater for all cultivars of cherries than for any of these apple cultivars. Information similar to that in Table 1, but for all sampling times, allows us to express the $99 \%$ mortality point in terms of the concentration at any particular time point. Table 5 gives this information for the cherry disinfestation data that are reported in Maindonald et al. (1992), as well as for the present data. Note that the $99 \%$ lethal concentration is higher for cherries than that for apples, for all time points. Cherries are much smaller than apples, with the spaces between fruit smaller than between apples. These smaller spaces may impede the circulation of the fumigant. Whatever the reason, the concentration-mortality relationship for apples is different from that for cherries.

The $\mathrm{LC}_{99}$ presented in this paper applies to all apple cultivars. For any new cultivar, estimation of an $\mathrm{LD}_{99}$ requires only the new sorption measurements that will allow the calibration of the $\mathrm{ct}_{\text {sum }}$ against injected dose. This conclusion offers a simplified approach for comparing different cultivars of the same fruit for development of fumigation treatment conditions that will be effective in killing codling moth potentially infesting export fruit. This overall analysis demonstrates the benefits, which (as in Maindonald et al., 1992) may be available from bringing together into one analysis the whole body of relevant data.

These results are important to the development of quarantine disinfestation procedures where historically, different cultivars of fruit have been treated as different entities in the development of quarantine disinfestation procedures. More recently, comparisons between different cultivars of the same fruit have been used to justify grouping two or more cultivars together. Maindonald et al. (1992) demonstrated that amalgamation of cherry cultivars is valid and desirable for analyses based on measures of actual $\mathrm{MeBr}$ concentration. Above, we have carried this result over to apples, showing that it is valid to amalgamate $\mathrm{ct}_{\text {sum }}$-mortality responses from the seven different apple 
Table 2

Slopes and intercepts, in the regression of $\mathrm{ct}_{\text {sum }}$ on methyl bromide dose for each apple cultivar ${ }^{\mathrm{a}}$

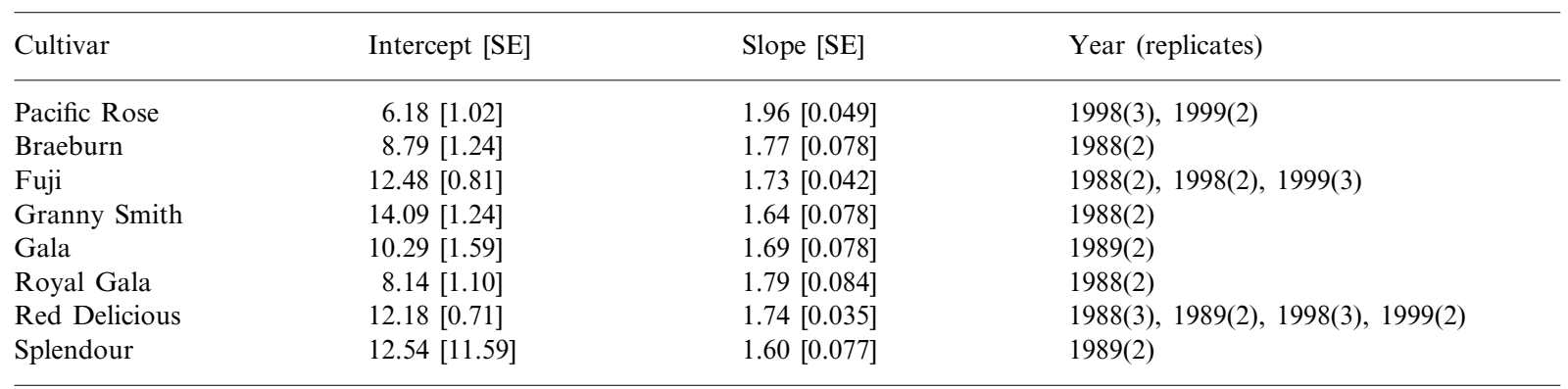

${ }^{a}$ The tabulated values are for 1988. For 1989 add 2.9 [SE 1.0] to the intercept, for 1998 subtract 0.02 [SE 0.68 ] and for 1999 add 0.2 [SE 0.74]. There were six doses and hence six values of $\mathrm{ct}_{\text {sum }}$ per replicate.

cultivars for which we had mortality data. Yokoyama et al. (1990) reached similar conclusions in their examination of three cultivars of nectarines.

Maindonald et al. (1992) detected differences in sorption patterns between cherry cultivars following $\mathrm{MeBr}$ fumigation. The present paper has demonstrated comparable differences in sorption patterns between apple cultivars. For acceptance of the existing treatment regime for a new variety, it is sufficient to demonstrate that, for the injected dose of $24 \mathrm{~g} \mathrm{~m}^{-3}$ that is used for currently approved treatments, the $\mathrm{ct}_{\text {sum }}$ is maintained at levels which lie within or above the range found for existing varieties. The provision of this reduced testing provides reassurance that an approved treatment will be effective when used with otherwise untested cultivars. This dose will control codling moth eggs potentially infesting any of the cultivars considered, including 'Pacific Rose'.

\section{Acknowledgements}

We wish to thank ENZAFRUIT New Zealand (International) for funding this study and Patrick Connolly (HortResearch) for assistance with graphical presentations.

\footnotetext{
Appendix A. Biological Mechanisms which may lead to a complementary log-log model. Derivation of the complementary log-log model.
}

A result from the theory of extreme values motivates the complementary $\log -\log$ model. These arguments explain why a complementary $\log -\log$ model may sometimes be appropriate. The understanding of mechanisms is typically inadequate to justify the use of this (or any other) model in any specific instance. The final justification for the particular choice of model must come from examination of the data.

Suppose that the organism dies when any one of a number $n$ of sites, which respond independently, becomes inactive because the level $Z$ of some quantity exceeds a threshold $z_{0}$. We define $p_{0}=1-F\left(z_{0} ; \theta\right)$, where $\theta$ is the expected value of $Z$, to be the probability that $Z$ reaches a level of $z_{0}$ or greater at any individual site. Take $Z_{(n)}$ to be

\section{Table 3}

Estimates and confidence intervals for the $\mathrm{ct}_{\text {sum }}$ when the methyl bromide dose is $24 \mathrm{~g} \mathrm{~m}^{-3}$, for each of the eight apple cultivars $^{\mathrm{a}}$

\begin{tabular}{llcc}
\hline Cultivar & $\mathrm{ct}_{\text {sum }}\left(\mathrm{g} \mathrm{h} \mathrm{m}^{-3}\right)$ & $\mathrm{SE}$ & $95 \%$ C.I. \\
\hline Pacific Rose & 53.3 & 1.0 & $51.3-55.3$ \\
Braeburn & 51.2 & 1.2 & $48.7-53.7$ \\
Fuji & 54.0 & 0.8 & $52.4-55.6$ \\
Granny Smith & 53.4 & 1.2 & $51.0-55.9$ \\
Gala & 50.8 & 1.6 & $47.7-54.0$ \\
Royal Gala & 51.0 & 1.4 & $48.3-53.7$ \\
Red Delicious & 54.0 & 0.7 & $52.5-55.5$ \\
Splendour & 50.9 & 1.6 & $47.7-54.1$ \\
\hline
\end{tabular}

\footnotetext{
a The tabulated estimates are for 1988. For 1989, the values should be increased by 2.9 [SE 1.0]. Estimates for 1998 and 1999 are statistically indistinguishable from those for 1988.
} 
Table 4

$\mathrm{LD}_{50}$ and $\mathrm{LD}_{99}$ estimates for 1-day old codling moth eggs infesting apples calculated using injected methyl bromide dose (g $\mathrm{m}^{-3}$ )

\begin{tabular}{|c|c|c|c|c|c|}
\hline Cultivar & $n^{\mathrm{a}}$ & $\mathrm{LD}_{50}{ }^{\mathrm{b}}$ & 95\% C.I. & $\mathrm{LD}_{99}{ }^{\mathrm{b}}$ & 95\% C.I. \\
\hline Pacific Rose ${ }^{c}$ & - & 20.4 & $18.5-22.2$ & 30.0 & $27.8-32.3$ \\
\hline Fuji & 14497 & 19.5 & $17.8-21.2$ & 30.5 & $28.3-32.6$ \\
\hline Gala & 13628 & 21.3 & $17.8-24.7$ & 32.5 & $28.1-36.9$ \\
\hline Granny Smith & 17011 & 19.6 & $16.4-22.7$ & 31.1 & $26.9-35.4$ \\
\hline Splendour & 24182 & 21.1 & $17.5-24.6$ & 32.9 & $28.3-37.6$ \\
\hline
\end{tabular}

\footnotetext{
a Includes non treated controls.

${ }^{\mathrm{b}}$ Values obtained by back-calibration from the $\mathrm{LC}_{50}$ and $\mathrm{LC}_{99}$ estimates for the $\mathrm{ct}_{\text {sum }}\left(\mathrm{g} \mathrm{h} \mathrm{m}^{-3}\right)$. Intercepts and slopes for the calibration line are given in Table 2 .

${ }^{\mathrm{c}}$ Codling moth eggs were not used in association with this cultivar.
}

the maximum of the $Z$ 's, over all sites. Then, extreme value theory (Mann and Singpurwalla, 1982) shows that the limiting distribution of $Z_{(n)}$ as $n$ becomes large takes just one of three 'canonical' forms. The form that is important for present purposes arises when $F(z)$ belongs to a class of distributions which includes the Normal and the Exponential. The probability that the maximum $Z_{(n)}$ will exceed $z_{0}$ is then, to arbitrary accuracy by taking $n$ large enough, given by the extreme value

$$
\begin{aligned}
p & =\operatorname{Pr}\left\{Z_{(n)} \geq z_{0}\right\} \\
& =1-\exp \left(-\exp \left(-\frac{\left(z_{0}-\gamma-\theta\right)}{\tau}\right)\right)
\end{aligned}
$$

where $\gamma$ and $\tau$ are functions of $n$, determined from the distribution function of $Z$.

In our application of Eq. (A.1), $n, \gamma, \tau$ and $z_{0}$ are thus all (unknown) constants. Setting $B=\tau-$ 1 and $A=-\left(z_{0}-\gamma\right) / \tau$, Eq. (A.1) may be rewritten

$p .=\operatorname{Pr}\left\{Z_{(n)} \geq z_{0}\right\}=1-\exp (-\exp (A+B \theta))$

or

$\log (-\log (1-p))=.A+B \theta$

Finally, we must relate $\theta$, which is the expected value of $Z$, to the dose $x$ or to the time $t$. If for example heat has destroyed the integrity of crucial membranes, so that there is steady leakage, $\theta$ may be directly proportional to time $t$. If there is leakage of the applied chemical, $\theta$ may be proportional to dose $x$. It then follows that for suitable $a$ and $b$ we can replace $A+B \theta$ by $a+b x$, and Eq. (A.2) becomes the complementary $\log -\log \operatorname{link}$ function

$\log (-\log (1-p))=.A+B \theta=a+b x$

With $x=$ time and setting $A=b e^{a}$, the hazard rate, which measures the instantaneous risk of death at time $x$ given survival to that time, is $A e^{b x}$. Thus $b=0$ indicates constant hazard.

Table 5

$99 \%$ mortality points $\left(\mathrm{g} \mathrm{m}^{-3}\right)$, for concentrations at individual time points ${ }^{\mathrm{a}}$

99\% Mortality point

\begin{tabular}{lll}
\cline { 2 - 3 } & Apples & Cherries \\
\hline $\mathrm{ct}_{\text {sum }}$ & $65.2 \mathrm{~g} \mathrm{~h} \mathrm{~m}^{-3}$ & $87.1 \mathrm{~g} \mathrm{~h} \mathrm{~m}^{-3}$ \\
$c_{5}$ & $33.6 \mathrm{~g} \mathrm{~m}^{-3}$ & $50.8 \mathrm{~g} \mathrm{~m}^{-3}$ \\
$c_{10}$ & $34.0 \mathrm{~g} \mathrm{~m}^{-3}$ & $50.3 \mathrm{~g} \mathrm{~m}^{-3}$ \\
$c_{30}$ & $33.1 \mathrm{~g} \mathrm{~m}^{-3}$ & $46.4 \mathrm{~g} \mathrm{~m}^{-3}$ \\
$c_{60}$ & $32.3 \mathrm{~g} \mathrm{~m}^{-3}$ & $42.4 \mathrm{~g} \mathrm{~m}^{-3}$ \\
$c_{90}$ & $32.3 \mathrm{~g} \mathrm{~m}^{-3}$ & $42.0 \mathrm{~g} \mathrm{~m}^{-3}$ \\
$c_{120}$ & $31.4 \mathrm{~g} \mathrm{~m}^{-3}$ & $38.8 \mathrm{~g} \mathrm{~m}^{-3}$
\end{tabular}

${ }^{\text {a }}$ For comparison we give the equivalent mortality points for the cherry disinfestation data from Maindonald et al. (1992). 


\section{References}

Finney, D.J., 1971. Probit analysis, Third ed. Cambridge University Press, London.

Longford, N.T., 1993. Random Coefficient Models. Oxford University Press, New York.

Maindonald, J.H., 1984. Statistical Computation. Wiley, New York.

Maindonald, J.H., Waddell, B.C., Birtles, D.B., 1992. Response to methyl bromide fumigation of codling moth (Lepidoptera: Tortricidae) eggs on cherries. J. Econ. Entomol. 85, 1222-1230.

MathSoft, 1999. S-PLUS Guide to Statistics, Volume 1. Data Analysis Products Division, MathSoft, Seattle, WA.
Mann, N.R., Singpurwalla, N.D., 1982. Extreme-value distributions. In: Kotz, S., Johnson, N.L., Read, C.B. (Eds.), Encyclopedia of Statistical Sciences, vol. 2. Wiley, New York, pp. 606-611.

McCullagh, P., Nelder, J.A., 1989. Generalized Linear Models. Chapman and Hall, London.

Tebbets, J.S., Hartsell, P.L., Nelson, H.D., Tebbets, J.C., 1983. Methyl bromide fumigation of tree fruits for control of the Mediterranean fruit fly: concentrations, sorption, and residues. J. Agric. Food Chem. 31, 247-249.

Yokoyama, V.Y., Miller, G.T., Hartsell, P.L., 1990. Evaluation of a methyl bromide quarantine treatment to control codling moth (Lepidoptera: Tortricidae) on nectarine cultivars proposed for export to Japan. J. Econ. Entomol. 83, $466-471$. 